\title{
Cognitive and adaptive outcomes and age at insult effects after non-traumatic coma
}

\author{
R J Forsyth, C P Wong, T P Kelly, H Borrill, D Stilgoe, S Kendall, J A Eyre
}

\begin{abstract}
Cognitive and adaptive behavioural outcome were studied in the identified survivors of a population based study of non-traumatic coma (NTC) in childhood. Children were assessed early (six weeks) and late (12 months) after NTC. At least $7 \%$ of those children in whom no suspicions of prior neurodevelopmental morbidity existed showed moderate or severe disability following NTC. Children over 2 years of age at insult showed some improvement between early and late assessments; however, children below 2 years showed no improvement. Differing age at insult effects were observed between aetiological groups. A relation between early age at first insult and poor outcome was particularly evident among children experiencing NTC caused by epilepsy. (Arch Dis Child 2001;84:200-204)
\end{abstract}

Keywords: coma; non-traumatic coma; prognosis; age related effects

Non-traumatic coma (NTC) is an important cause of morbidity and mortality in children. In the first population based epidemiological survey of paediatric NTC in the UK we showed an incidence of 30.8 episodes per 100000 children under 16 per year. ${ }^{1}$ Episodes were defined on the basis of either a Glasgow Coma Score of less than 12 for more than six hours; or death before hospital admission associated with coma (see Wong et al for further details of eligibility and exclusion criteria $^{1}$ ). Overall series mortality was $46 \%$, with aetiology specific mortality rates ranging from $3 \%$ to $84 \%$. In this paper we address aspects of cognitive and adaptive outcome among survivors.

In recent years there has been renewed interest in age at insult effects in childhood acquired brain injury. ${ }^{23}$ Classic studies in subhuman primates by Kennard showed a correlation between young age at insult and good motor outcome. ${ }^{4}$ These have, in part, been responsible for a general belief that children make better recoveries from acquired brain insult than adults. ${ }^{3}$ However, in the context of traumatic brain injury it is now clear that this "Kennard principle" is fallacious. ${ }^{25}$ Brain injury impacts on new learning, and can disturb the entire developmental trajectory in the period remaining between the injury and attaining maturity. ${ }^{67}$ The younger the age at injury, the longer the period potentially available for these new learning effects to act. For example, in relation to traumatic brain injury, late cognitive outcomes (particularly relative to age matched peers) are poorer the younger the age at injury and the greater the time post-injury. ${ }^{7}$ Detailed aspects of age at insult effects appear to vary with the precise nature of the insult. ${ }^{2}$ We examined age at insult effects following non-traumatic coma.

\section{Methods}

RECRUITMENT

The background to the study has been described in detail elsewhere. ${ }^{1}$ In brief, the study population comprised children (between 1 month and 15 years 11 months of age) experiencing episodes of NTC between July 1994 and December 1995 in the former Northern NHS region of England.

The primary aetiology of the encephalopathic episode was determined after inspection of medical and/or postmortem data by one researcher (CPW) and assigned to one of eight general aetiological categories. ${ }^{1}$ These were (in decreasing order of frequency): infections; unknown (including suspected but unproven aetiologies); intoxication; epilepsy (complications of seizures or their treatment); congenital (complications of congenital malformations); others; accidental (for example, inadvertent strangulation); and metabolic processes.

\section{OUTCOME ASSESSMENT}

Nominal follow up time points were six weeks and 12 months after admission. All children were assessed by a formal clinical neurological examination and by screening for visual and auditory deficits. One investigator (CPW) performed this assessment. In addition a cognitive assessment was performed by HB or DS using age appropriate psychometric instruments. In children below 3.5 years, we assessed cognitive function using the Bayley Scales of Infant Development. ${ }^{10}$ Between 3.5 and 6.0 years, we used the WPPSI- $\mathrm{R}^{\mathrm{UK}},{ }^{11}$ and above 6 years the WISC-III ${ }^{\mathrm{UK}} .12$

Clinical neurological assessment included cranial and peripheral motor and sensory neurological examination, including cerebellar function. Visual outcome has been reported separately. ${ }^{9}$

\section{AGE AT INSULT ANALYSES}

To look for effects of age at insult across the entire age range of the study population we needed to generate a consolidated all age "cognitive quotient" (CQ). We consolidated the Bayley Mental Developmental Index (MDI), WPPSI full scale IQ, and WISC full scale IQ into a single all age CQ. All have a standard scoring format of mean of 100 and standard deviation of 15 . 
Table 1 Summary of cognitive outcomes

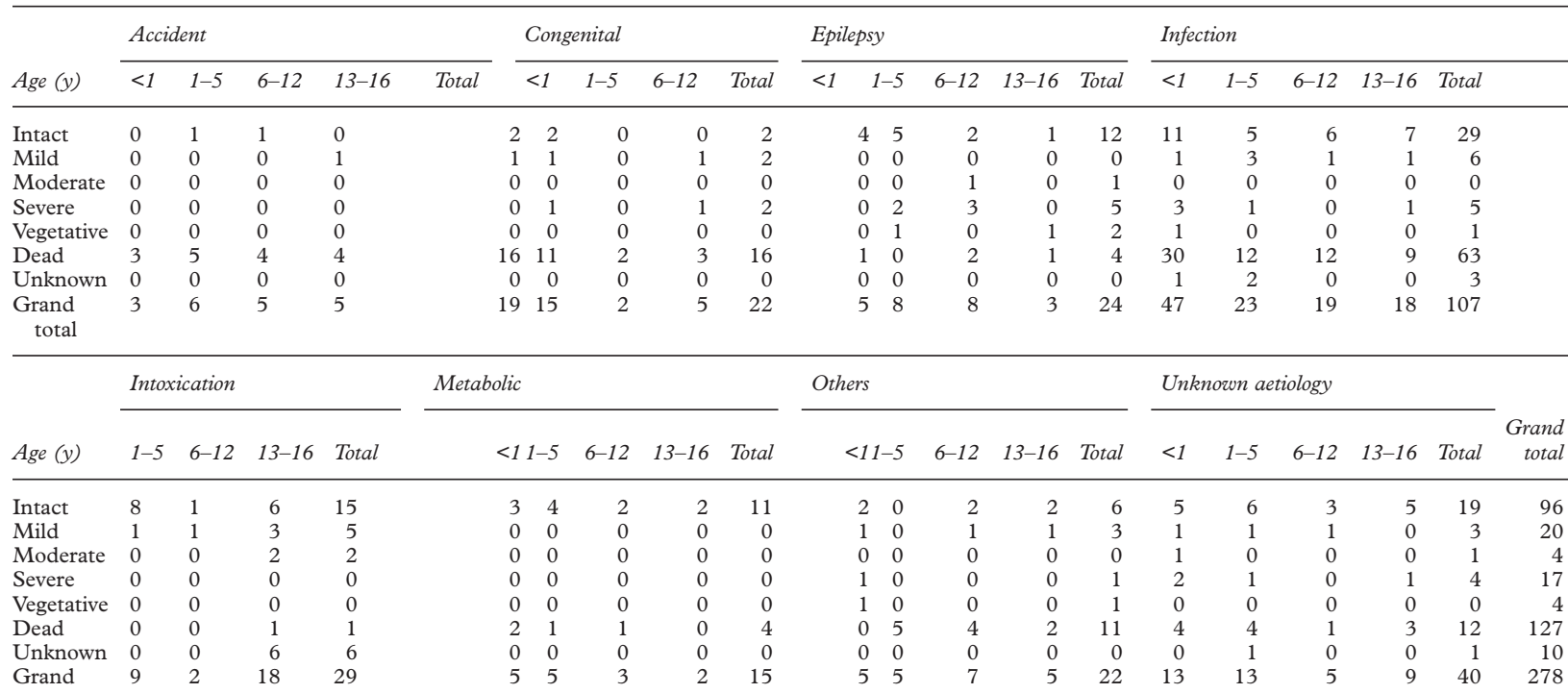

Late cognitive outcomes (at nominal 12 month time point) for entire series of 287 children, analysed by age band and aetiological category. Psychological outcomes are defined as follows. "Intact": Cognitive quotient (CQ, see text) $\geqslant 85$ or thought no change relative to premorbid functioning. "Mild": CQ 70-84 and/or mild behavioural disturbances. "Moderate": CQ 55-69 and/or moderate behavioural disturbances. "Severe": CQ < 55 and/or severe behavioural disturbances. "Vegetative": untestable.

There were no infants in the intoxication group.

\section{ADAPTIVE BEHAVIOUR}

Logistical constraints prevented the full assessment of adaptive behaviour in all survivors of NTC. However, if any impairment was identified in any domain (neurological, cognitive, or special sensory), a single assessor (SK) assessed the child's adaptive behaviour using the Vineland Adaptive Behaviour Scales (VABS). This provides a normalised score aggregating performance in four domains: communication, daily living skills, socialisation, and motor skills. ${ }^{13}$

If a child with known prior neurological disability was considered by care givers to have returned to prior levels of disability, and if detailed neurological and special sensory examination revealed no new impairments not already identified, then we regarded the child as having an intact outcome. We did not perform an adaptive behaviour assessment under these circumstances, as it would not reflect the consequences of the NTC.

As VABS assessments were confined to children with identified neurological, sensory, or cognitive/behavioural impairments, we assumed that a child with no identifiable impairments in these domains would have a normal range adaptive behaviour profile. Consequently, we grouped children into adaptive behaviour bands, with analysis based on the resulting categorical, rather than continuous, data. Normalised Vineland adaptive behaviour composite scores were categorised as normal range (adaptive behaviour composite score within two standard deviations of mean; $\geqslant 70$ ); moderately disabled ( $55 \leqslant$ adaptive behaviour composite $<70$ ); or severely disabled (adaptive behaviour composite <55).

PREMORBID DISABILITY

For some analyses of the consequences of NTC among survivors, we needed to identify and exclude children with known or suspected premorbid neurodisability. As part of the assessment procedure, we asked health visitors and general practitioners to complete a structured questionnaire indicating the child's premorbid condition. Additionally, we scrutinised general practitioner records for evidence of premorbid conditions that could have had implications for the child's cognitive development. We excluded children from these analyses if there was evidence of premorbid cognitive deficit, of conditions such as epilepsy or head injury which could impair cognitive function, or of provision under UK educational procedures to meet an identified special educational need.

STATISTICAL METHODS

Analyses used $\chi^{2}$, Fisher exact, $t$ test, and nonparametric correlation techniques. Significance was assumed at $\mathrm{p}<0.05$.

\section{Results}

A total of 283 episodes of NTC meeting study criteria were identified in 278 children (for further details see Wong and colleagues ${ }^{1}$ ). Of the three children multiply recruited, one had four episodes of status epilepticus during the study period; another had two episodes. Both survived to follow up. A third child suffered two periods of NTC resulting from complications of congenital heart disease, and died. Outcome data are therefore expressed relative to a denominator of 278 children. A total of 127 children died: 59 before hospital admission identified through OPCS returns, 66 hospital deaths, and two late deaths. Thus 151 children survived to late follow up and were potentially available for assessment.

We made extensive efforts to contact survivors, delaying the median late follow up interval from a nominal 12 months to 13 months 
Table 2 Aetiologies among "prior normal" group

\begin{tabular}{|c|c|c|c|c|c|c|c|c|c|}
\hline & Accident & Congenital & Epilepsy & Infection & Intoxication & Metabolic & Others & Unknown & Totals \\
\hline Prior normal survivors & $3(2.6 \%)$ & $3(2.6 \%)$ & $12(10.4 \%)$ & $36(31.3 \%)$ & $20(17.4 \%)$ & $11(9.6 \%)$ & $6(5.2 \%)$ & $24(20.9 \%)$ & $115(100 \%)$ \\
\hline Other survivors & $0(0 \%)$ & $3(8.3 \%)$ & $8(22.2 \%)$ & $8(22.2 \%)$ & $8(22.2 \%)$ & $0(0 \%)$ & $5(13.9 \%)$ & $4(11.1 \%)$ & $36(100 \%)$ \\
\hline Died & $16(12.6 \%)$ & $16(12.6 \%)$ & $4(3.2 \%)$ & $63(49.6 \%)$ & $1(0.8 \%)$ & $4(3.2 \%)$ & $11(8.7 \%)$ & $12(9.4 \%)$ & $127(100 \%)$ \\
\hline Totals & $19(6.8 \%)$ & $22(7.9 \%)$ & $24(8.6 \%)$ & $107(38.5 \%)$ & $29(10.4 \%)$ & $15(5.4 \%)$ & $22(7.9 \%)$ & $40(14.4 \%)$ & $278(100 \%)$ \\
\hline
\end{tabular}

Distribution of aetiologies among "prior normal" group compared to remainder of study cohort: other survivors and deaths. Table cells show numbers with row percentages in brackets. See text.

(range 8-37 months). Neurological, special sensory, and cognitive assessments were completed in 141 children ( $93 \%$ of survivors). Of the remainder, six families declined to participate, and four children were untraceable. Intoxication was the commonest aetiology in the group lost to follow up. ${ }^{1}$ Table 1 presents a summary of the cognitive outcome data.

PREMORBID DISABILITY

We identified 115 children among the 151 survivors in whom prior neurocognitive morbidity was considered unlikely by the stringent criteria above. There was no significant difference in mean age between this group and the remainder of the 278 child cohort ( $p>0.8$; unpaired $t$ test), but there were significant differences in the frequencies of specific aetiologies between these prior normal children and the full cohort (see table 2). Relative to the series as a whole (deaths plus other survivors), intoxication and unknown aetiologies were over represented, and infection under represented in the prior normal survivors group $\left(\mathrm{p}<0.0001 ; \chi^{2}=48.7 ; \mathrm{df}=7\right.$; table 2$)$.

AGE AT INSULT AND COGNITIVE OUTCOMES We analysed the 115 children in the prior normal subset for age at insult effects. No relation was evident between age at insult and final CQ for the 115 children as a whole (Pearson's $r=0.03 ; \mathrm{p}>0.5)$.

Separate analyses were performed within each aetiological group across the entire age range. We found no significant correlation between age at insult and final CQ, nor were there significant gender effects.

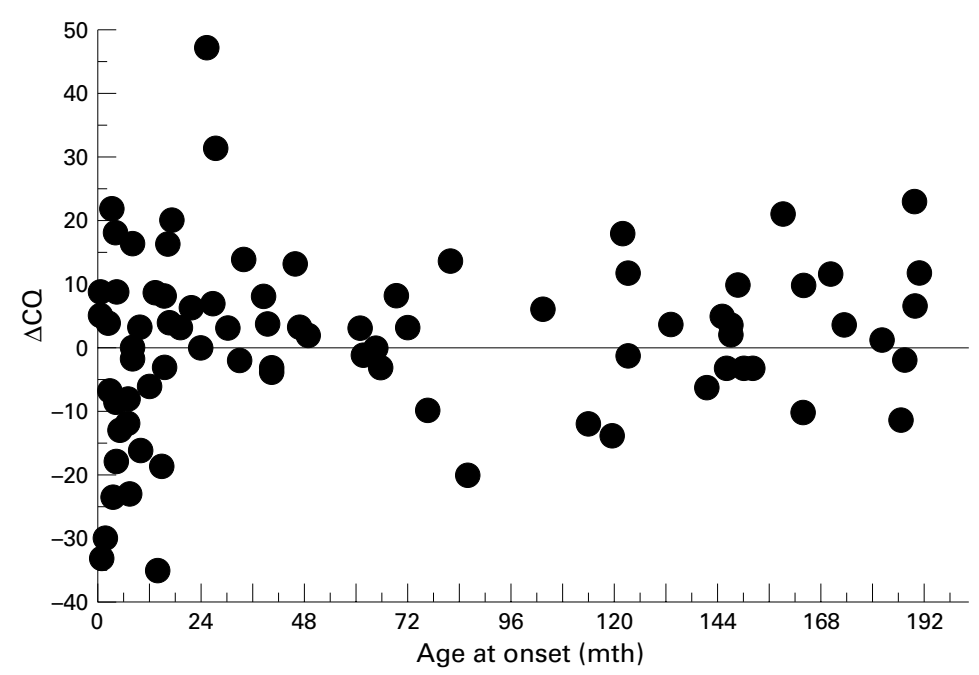

Figure 1 Effect of age at injury on difference in CQ between early (six week) and late (12 month) time points ( $\triangle C Q)$.
TIME COURSE OF RECOVERY AFTER NTC

Cognitive data were available at both early and late time points (nominally six weeks and 12 months post-insult) for 82 of the 115 prior normal children. This gave an opportunity to assess the recovery at two time points. For a small number of children who crossed age limits for the use of the cognitive outcome instruments between first and second assessments, we needed to use a different instrument at each time point. Bayley, WPPSI, or WISC data were consolidated into a single all age CQ (see Methods). Across the whole age range, there was no correlation between age (treated as a continuous variable) and change in CQ between early and late time points $(\Delta \mathrm{CQ})$. However, we noted a trend for younger children not to show the improvement between early and late time points shown by older children. For children over 24 months at insult, mean $\triangle \mathrm{CQ}$ was significantly greater than zero (mean $+4.1 ; 95 \%$ confidence interval (CI) 0.9 to $7.4 ; \mathrm{p}<0.05$ ), implying some spontaneous recovery between early and late time points. This was not the case for children less than 24 months at insult who showed a mean $\triangle \mathrm{CQ}$ of $-3.3(95 \% \mathrm{CI}-9.0$ to +2.3 ; $\mathrm{p}>0.2)$, implying no significant spontaneous recovery in cognitive abilities (see fig 1 and Discussion).

Subgroup analysis by aetiological category showed a significant relation between $\triangle C Q$ and age at insult for the epilepsy group (Spearman $r$ corrected for ties 0.61 ; two tailed $\mathrm{p}<0.05$ ). Previously normal children experiencing an episode of NTC caused by epilepsy under the age of 12 months showed a mean fall in CQ in the interval six weeks to 12 months insult of -12.4 (SEM 2.0) points ( $\mathrm{p}<0.05)$. This fall is additional to the morbidity already evident six weeks after insult.

ADAPTIVE BEHAVIOUR

Of 151 late survivors, 141 had neurological and cognitive assessments. Ninety three children showed no impairment in any assessed domain. These children were thus assigned an assumed normal range VABS adaptive behaviour composite score category. A remaining 48 were eligible for full Vineland assessment, of whom we assessed 33 (see table 3). We found significant morbidity in the adaptive behaviour domain, with $13 \%$ of survivors showing an adaptive behaviour composite score more than two standard deviations below the population mean (that is, in the moderate or severe categories as described above). Among the prior normals, eight of 115 children $(7.0 \%)$ showed this degree of disability.

There was a significant association between the presence of significant disability after an episode of NTC (defined as adaptive behaviour 
Table 3 Adaptive behaviour outcome category

\begin{tabular}{lclllr}
\hline & Normal range & $\begin{array}{l}\text { Moderate } \\
\text { disability }\end{array}$ & $\begin{array}{l}\text { Severe } \\
\text { disability }\end{array}$ & $\begin{array}{l}\text { Missing or lost to } \\
\text { follow up }\end{array}$ & \multicolumn{1}{c}{ Totals } \\
\hline Prior normals & $95^{\star}(83)$ & $2(1.7)$ & $6(5.2)$ & $12(10.4)$ & $115(100)$ \\
Other survivors & $13 \dagger(36)$ & $2(5.6)$ & $8(22.2)$ & $13(36.1)$ & $36(100)$ \\
Total survivors & $108(71.5)$ & $4(2.6)$ & $14(9.3)$ & $25(16.6)$ & $151(100)$
\end{tabular}

Vineland Adaptive Behaviour Composite score graded as normal range $(\geqslant 70)$, moderate disability $(55-69)$, or severe disability $(<55)$. Absolute numbers are shown, with row percentages in brackets. For the method of identification of the prior normal group, see text.

*Includes 82 presumed normal range adaptive behaviour results on the basis of lack of demonstrable neurological, cognitive, or special sensory impairment (see text).

tIncludes 11 presumed normal range adaptive behaviour results on the basis of lack of demonstrable neurological, cognitive, or special sensory impairment (see text).

of less than -2SD) and the presence of premorbid disability. Children identified as having significant disabilities were under represented in the prior normal group (table 3; $\mathrm{p}<0.0001$, Fisher exact test). This suggests that some of the adaptive behaviour morbidity seen in the series as a whole was indeed of premorbid origin (see Discussion).

\section{Discussion}

MEASUREMENT OF IMPACT OF DISABILITY

The quantification of neurological morbidity is particularly challenging in children. Adult measures of acquired neurological disability such as the Glasgow Outcome Scale and the Disability Rating Scale ${ }^{14-16}$ compare outcome to a baseline of mature independent adult functioning. In contrast, paediatric measures must relate to age dependent expectations of independence. Furthermore the neurological, cognitive, and other aspects of physiological child development resume after a neurological insult and can be modified as a consequence of its occurrence: thus the shortfall between current functional status and the changing age dependent standards of "normality" is dynamic. There is an additional dynamic relation between the component parts of the total morbidity burden. The relative contributions of the physical, behavioural, and cognitive subdomains to overall morbidity are highly variable. These complex interrelations hamper the creation of unidimensional global morbidity scores.

The conceptual framework of the World Health Organisation International Classification of Impairment, Disability, and Handicap $(\mathrm{ICIDH})^{17}$ is widely used. "Impairments" relate to limitations of function at the level of an individual organ while "disabilities" refer to limitations at the level of the individual's ability to function. Limitations in the child's ability to fulfil meaningful roles in society are designated within the WHO classification under the rubric of "handicap", although this remains unsatisfactory both as a term and a concept, ${ }^{18}$ and is replaced by the concept of "participation" in the forthcoming revision of the ICIDH. The neurological and cognitive/behavioural assessments we used can be regarded as measures of impairment or disability. They share with other measures of impairment the advantage of relative ease of assessment. The logistical implications of performing Vineland assessments in all survivors obliged us to use measures of impairment as a screening device, making the implicit assumption that "handicap" (as measured by the Vineland score) would not occur in the absence of detectable impairment in one or more of the assessed domains. We acknowledge the limitations of this approach. Additionally, the limitations of the Vineland instrument in measuring the full impact of an episode of NTC are recognised. For example, the Vineland instrument is an entirely "within the child" measure, that does not attempt to capture the effects of a child's illness on wider family function.

BURDEN OF DISABILITY CAUSED BY NTC

While no prior data were available, it seemed probable that some children with pre-existing neurodisabilities might be at increased risk for NTC of particular aetiologies. Table 2 confirms an association between certain aetiologies of NTC and the presence or otherwise of premorbid disability. While it was not possible to systematically collect data on premorbid functioning, we attempted to identify a cohort in whom there were no prior suspicions of particular neurocognitive difficulties.

The statistically significant association between diminished adaptive behaviour and the suspicion of premorbid disability (table 3 and Results) suggests that some of the adaptive behaviour morbidity seen in the series as a whole was indeed of premorbid origin. Nevertheless at least $7 \%$ of the stringently defined prior normal group acquired moderate or severe levels of adaptive disability, defined as VABS scores less than two standard deviations below population mean, as a direct result of their NTC. This figure excludes any disability among the group lost to follow up (table 3). Additionally, in this selected population of "prior normal" children the prior distribution of adaptive behaviour scores might be expected to be shifted to the right (more capable) relative to the full "normal" population. Thus this figure of $7 \%$ is an underestimate of the prevalence rate of moderate or severe disability that can be directly attributed to NTC.

AGE AT INJURY EFFECTS

It is now recognised that traumatic brain injury is an event that will alter the entire subsequent developmental trajectory for a child. As a child injured in the early years of life attempts to acquire new skills, the apparently subtle deficits in new learning that are often selectively impaired in TBI may compound over the years into a significant late morbidity. ${ }^{7}$ This study provides evidence for limited age at insult effects in NTC: they are obscured to a considerable extent by differences in the pathophysiology of the NTC between aetiological groups. Children recovering from NTC are probably a more heterogeneous group than their traumatically injured counterparts. Nevertheless it is clear that very young children (under 24 months of age at insult) are a group with a more guarded prognosis, showing little or no catch up over time after NTC (fig 1). This may reflect a limited repertoire of pre-existing skills on which to draw in making a recovery.

The relative weakness of the age related effects seen here may in part reflect the use of 
global IQ scores rather than specific tests of attentional, executive, and other functions that may be more selectively impaired following acquired brain injury. Additionally, effects may be obscured by the need to use different instruments within different age bands: it is not clear that the Bayley Mental Developmental Index is measuring precisely the same processes in infants that the WISC reflects in older children

Age related effects on outcome can reflect several different mechanisms. ${ }^{2}$ Our study, assessing at a standard interval since insult, highlights age at injury relations. These may reflect the consequences of selective damage to brain regions undergoing active development at the time of injury. ${ }^{2}$ Additionally, the association of particular aetiologies with age ${ }^{1}$ will be important. Within the various aetiological categories, significant age at insult effects were only evident within the epilepsy group, with younger children having a poorer outlook. Epileptic encephalopathies presenting in early childhood are known to have a poorer long term outlook. ${ }^{19}$

By comparison, time since insult analyses may highlight the consequences of damage to structures required for skill acquisition. Cross sectional studies have shown that cognitive weaknesses among children with early brain insults caused by hydrocephalus ${ }^{20}$ or myelomeningocoele $^{21}$ are more evident in older than younger children. Figure 1 begins to show this effect longitudinally in children following NTC. It will be important to reassess this cohort at later timepoints.

We have highlighted significant morbidity following NTC in childhood, and provided further support for the hypothesis of increased vulnerability of very young brains to cognitive sequelae of acquired brain injury.

This study was supported by The Wellcome Trust and the former Northern Regional Health Authority.
1 Wong CP, Forsyth RJ, Kelly TP, Eyre JA. Incidence, aetiology and outcome of childhood non-traumatic coma: a prospective, population-based study. Arch Dis Child 2001;84:???????

2 Taylor HG, Alden J. Age-related differences in outcomes following childhood brain insults: an introduction and overview. F Int Neuropsychol Soc 1997;3:555-67.

3 Webb C, Rose FD, Johnson DA, Attree EA. Age and recovery from brain injury: clinical opinions and experimental evidence. Brain Inj 1996;10:303-10.

4 Kennard MA. Age and other factors in motor recovery from precentral lesions in monkeys. Am f Physiol 1936;115:138precen.

5 Schneider GE. Is it really better to have your brain lesion early: a revision of the "Kennard Principle". Neuropsychology 1979;17:557-83.

6 Anderson V, Moore C. Age at injury as a predictor of outcome following pediatric head injury: a longitudinal perspective. Child Neuropsychol 1995;1:187-202.

7 Fletcher JM, Ewing-Cobbs L, Francis DJ, Levin HS. Variability in outcomes after traumatic brain injury in
children: a developmental perspective. In: Broman SH, Michel ME, eds. Traumatic head injury in children. New York: Oxford, 1995:22-39.

8 James H, Trauner D. The Glasgow Coma Scale. In: James $\mathrm{H}$, Anas N, Perkin R, eds. Brain insults in infant and children. Pathophysiology and management. Orlando: Grune and Stratton, 1985:179-82.

9 Ziakas NG, Wong CP, Ramsay AS, et al. Visual impairment in children with acute non-traumatic coma. 7 Pediatr Ophthalmol Strabismus (in press).

10 Bayley N. Bayley's Scales of Infant Development, 2nd edn. New York: Psychological Corporation, 1993.

11 Wechsler D. WPPSI-R ${ }^{U K}$ manual. Weschsler Preschool and Primary Scales of Intelligence - revised. Sidcup, Kent: Psychological Corporation, 1989.

12 Wechsler D. WISC-IIIU manual-Wechsler Intelligence Scale for Children - III ${ }^{U K}$. Sidcup, Kent: Psychological Corporation, 1991.

13 Sparrow S, Balla DA, Cicchetti DV. Vineland Adaptive Behavior Scale: interview edition, expanded form manual. Circle Pines, MN: American Guidance Service, 1984.

14 Jennett $\mathrm{B}$, Bond $\mathrm{M}$. Assessment of outcome after severe brain damage. Lancet 1975;1:480-4.

15 Hall K, Cope DN, Rappaport M. Glasgow Outcome Scale and Disability Rating Scale: comparative usefulness in following recovery in traumatic head injury. Arch Phys Med Rehabil 1985;66:35-7

16 Rappaport M, Hall KM, Hopkins K, et al. Disability Rating Scale for severe head trauma: coma to community. Arch Phys Med Rehabil 1982;63:118-23.

17 WHO. International classification of impairments, disabilities, and handicaps. Geneva: World Health Organisation, 1980.

18 Hutchinson T. The classification of disability. Arch Dis Child 1995;73:91-3.

19 Aicardi J. Epilepsy in childhood, 2nd edn. Philadelphia: Lippincott-Raven, 1997.

20 Dennis M, Hendrick EB, Hoffman HL, Humphreys RP. Language of hydrocephalic children and adolescents. F Clin Exp Neuropsychol 1987;9:593-621.

21 Wills K, Holmbeck G, Dillon K, McLone D. Intelligence and achievement in children with myelomeningeocele. $\mathcal{f}$ Pediatr Psychol 1991;15:161-76. 